\title{
Coherent Observers for Linear Quantum Stochastic Systems *
}

\author{
Zibo Miao $^{\mathrm{a}, \mathrm{b}}$, Matthew R. James ${ }^{\mathrm{a}}$, Ian R. Petersen ${ }^{\mathrm{c}}$ \\ ${ }^{a}$ ARC Centre for Quantum Computation and Communication Technology, Research School of Engineering, Australian \\ National University, Canberra, ACT 2601, Australia \\ ${ }^{\mathrm{b}}$ Department of Electrical and Electronic Engineering, University of Melbourne, Melbourne, VIC 3010, Australia \\ ${ }^{\mathrm{c}}$ School of Information Technology and Electrical Engineering, University of New South Wales at the Australian Defence \\ Force Academy, Canberra, ACT 2600, Australia
}

\begin{abstract}
The theory of observers is a basic part of classical linear system theory. The purpose of this paper is to develop a theory of coherent observers for linear quantum systems. We provide a class of coherent quantum observers, which track the observables of a linear quantum stochastic system in the sense of mean values, independent of any additional quantum noise in the observer. We prove that there always exists such a coherent quantum observer described by quantum stochastic differential equations in the Heisenberg picture, and show how it can be designed to be consistent with the laws of quantum mechanics. We also find a lower bound for the mean squared estimation error due to the uncertainty principle. In addition, we explore the quantum correlations between a linear quantum plant and the corresponding coherent observer. It is shown that considering a joint plant-observer Gaussian quantum system, entanglement can be generated under the condition that appropriate coefficients of the coherent quantum observer are chosen, and this issue is illustrated in an example. These results pave the way towards observer-based quantum control.
\end{abstract}

Key words: Quantum stochastic differential equations, coherent quantum observers, quantum correlations.

\section{Introduction}

Effective control requires that sufficient information about the target plant is available to the controller. However, in many situations, full knowledge of the plant is not accessible and unknown quantities may be estimated on the basis of the available information (Ellis (2002), Yamamoto (2006)). It is well established classically that the Kalman filter, which computes the conditional expectations of the state variables of the plant, is a statistical approach to state estimation based on dynamical linear-Gaussian models (Anderson and

\footnotetext{
ऋ We gratefully acknowledge support by the Australian Research Council Centre of Excellence for Quantum Computation and Communication Technology (project number CE110001027), Australian Research Council Discovery Project (project numbers DP110102322 and DP120101549) and the Air Force Office of Scientific Research (grant numbers FA2386-09-1-4089, FA2386-12-1-4075 and FA2386-121-4084).

Email addresses: Zibo.Miao@anu.edu.au (Zibo Miao), Matthew. James@anu.edu. au (Matthew R. James), I.Petersen@adfa.edu.au (Ian R. Petersen).
}

Moore (1979)). Likewise, in the emerging field of coherent quantum control, information on the quantum plant is also needed for efficacious control (Wiseman and Milburn (2010)). When the quantum plant is continuously monitored, the Belavkin-Carmichael quantum filter may be used to compute conditional expectations of plant variables (Belavkin (1994), Bouten et al. (2007), Wiseman and Milburn (2010)). Mathematically, the quantum filter computes a quantum conditional expectation onto a commutative subspace of output signals generated by measurement processes. In the special case of linear quantum stochastic systems, i.e. those for which certain conjugate variables evolve linearly in the Heisenberg picture (as in linear quantum optics; e.g., see Wiseman and Milburn (2010), James et al. (2008)), the quantum filter reduces to the Kalman filter.

As a step towards better understanding of fully quantum non-commutative estimation and control, in this paper we extend Luenberger's approach (Luenberger (1966)) for observer design to linear quantum stochastic system$\mathrm{s}$, whose dynamics are described by linear quantum stochastic differential equations (QSDEs). In classical linear systems theory, an observer is a system driven by 
the plant outputs (and control inputs where appropriate) and designed in such a way that the observer variables asymptotically track the plant variables. As is well known, if the plant is detectable, then the observer gain may be chosen so that the estimation error exponentially converges to zero.

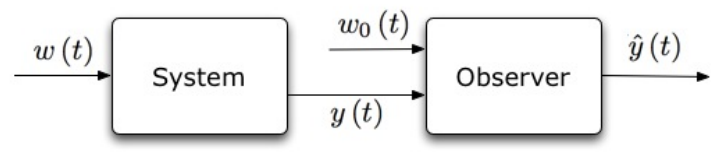

Fig. 1. A plant-observer composite quantum system

In the quantum case we consider, as Fig. 1 shows, the output of the quantum plant is a fully quantum signal (e.g. an optical field), and no measurement is involved in this framework. This signal drives the observer, another fully quantum system which tracks the quantum plant asymptotically in the sense of mean values, in a series arrangement (Carmichael (1993), Gough and James (2009)). Since the algebraic equations corresponding to a direct analog of the classical Luenberger observer need not correspond to a quantum physical system, here we have to consider the existence of such an observer constrained by physical realisability conditions distinguished from the classical case. We find explicit forms for the coefficients of a physically realisable coherent observer, and prove that there always exists a coherent quantum observer. This may involve including additional quantum noise in the observer (James et al. (2008), Miao and James (2012)). It is worth noting that the quantum observer we propose in this paper "observes" the observables of a linear quantum plant coherently, but not the quantum states. Here we mention another publication on quantum observers (Yamamoto (2006)), where the observers considered are classical systems, and measurement is involved.

In this paper, we give a full and general theorem with detailed proofs concerning the existence of coherent quantum observers, whereas only a partial result is presented in Miao and James (2012). New results for a simplified observer design for annihilation-operator linear quantum systems are included, and we show an explicit structure for the observer in this situation without additional quantum noise. Furthermore, we provide the fundamental limit for the mean squared estimation error by using a coherent observer. Observations in our work regarding quantum correlations in joint plant-observer quantum systems suggest there are inherent differences between tracking systems with classical and quantum components, making this topic not only practical but also interesting. Applications of coherent quantum observers can be expected, as the notion of an observer and estimate that we use has a history of success in the classical literature (Luenberger (1966)). We have applied the observer theory to fully quantum coherent tracking feed- back control in Miao et al. (2015). Moreover, quantum observers design using stronger criteria involving covariances have been developed in Miao et al. (2015).

The paper is organised as follows. Section 2 presents the linear quantum stochastic systems of interest as well as the physical realisability conditions. In Section 3, we give a detailed proof of the existence of coherent quantum observers, and provide a lower bound for the mean squared observer error. This is followed by Section 4, in which we analyse the quantum correlations in a joint plant-observer Gaussian system, including quantum discord. Section 5 provides some concluding remarks and future research directions.

Notation. In this paper, the asterisk is used to indicate the Hilbert space adjoint $X^{\star}$ of an operator $X$, as well as the complex conjugate $z^{\star}=x-i y$ of a complex number $z=x+i y$ (here, $i=\sqrt{-1}$ and $x, y \in \mathbb{R}$ ). The conjugate transpose $A^{\dagger}$ of a matrix $A=\left\{a_{i j}\right\}$ is defined by $A^{\dagger}=\left\{a_{j i}^{\star}\right\}$. Also defined are the conjugate $A^{\sharp}=\left\{a_{i j}^{\star}\right\}$ and the transpose $A^{T}=\left\{a_{j i}\right\}$ matrices, so that $A^{\dagger}=\left(A^{T}\right)^{\sharp}=\left(A^{\sharp}\right)^{T}$. Real and imaginary parts of a matrix $A$ are denoted by $\Re(A)$ and $\Im(A)$ respectively. The mean value (quantum expectation) of an operator $X$ is denoted by $\langle X\rangle$. The commutator of two operators $X, Y$ is defined by $[X, Y]=X Y-Y X$. The anti-commutator of two operators $X, Y$ is defined by $\{X, Y\}=X Y+Y X$. The tensor product of operators $X, Y$ defined on Hilbert spaces $\mathrm{H}, \mathrm{G}$ is denoted $X \otimes Y$, and is defined on the tensor product Hilbert space $\mathrm{H} \otimes \mathrm{G}$. $I_{n}(n \in \mathbb{N})$ denotes the $n$ dimensional identity matrix. $0_{n}(n \in \mathbb{N})$ denotes the $n$ dimensional zero matrix.

\section{LINEAR QUANTUM STOCHASTIC SYS- TEMS}

The dynamics of an open quantum system are uniquely determined by the triple $(S, L, \mathcal{H})$ (Parthasarathy (1992), Gough and James (2009)). The self-adjoint operator $\mathcal{H}$ is the Hamiltonian describing the self-energy of the system. The unitary matrix $S$ is a scattering matrix, and the column vector $L$ with operator entries is a coupling vector. $S$ and $L$ together specify the interface between the system and the fields. Given an operator $X$ defined on the initial Hilbert space $\mathrm{H}$, its Heisenberg evolution is defined by

$$
\begin{aligned}
d X= & (\mathcal{L}(X)-i[X, \mathcal{H}]) d t+d W^{\dagger} S^{\dagger}[X, L] \\
& +\left[L^{\dagger}, X\right] S d W+\operatorname{tr}\left[\left(S^{\dagger} X S-X\right) d \Lambda_{w}\right],
\end{aligned}
$$

with

$$
\mathcal{L}(X)=\frac{1}{2} L^{\dagger}[X, L]+\frac{1}{2}\left[L^{\dagger}, X\right] L,
$$

which is called the Lindblad superoperator. The operators $W$ are defined on a particular Hilbert space called 
a Fock space F. When the fields (the number of fields is $\left.n_{w}\right)$ are in the vacuum states, these are the quantum Wiener processes which satisfy the Itô rule

$$
d W d W^{\dagger}=I_{n_{w}} d t
$$

Input field quadratures $W+W^{\sharp}$ and $-i\left(W-W^{\sharp}\right)$ are each equivalent to classical Wiener processes, but do not commute. A field quadrature can be measured using homodyne detection (Gardiner and Zoller (2000), Gough and James (2009)). The gauge processes $\Lambda_{w}$ are input signals to the system as well.

We assume there is no interaction between different fields, and thus hereafter we assume $S$ to be the identity matrix without loss of generality (James et al. (2008)). This assumption eliminates the first time on the right hand side of (1). To be specific,

$$
\begin{aligned}
d X= & (\mathcal{L}(X)-i[X, \mathcal{H}]) d t \\
& +\frac{1}{2}\left([X, L]-\left[X, L^{\dagger}\right]\right) d W_{1} \\
& -\frac{i}{2}\left([X, L]+\left[X, L^{\dagger}\right]\right) d W_{2},
\end{aligned}
$$

with

$$
\left[\begin{array}{l}
W_{1} \\
W_{2}
\end{array}\right]=\left[\begin{array}{c}
W+W^{\sharp} \\
-i\left(W-W^{\sharp}\right)
\end{array}\right] .
$$

The quadrature form of the output fields is given by

$$
\left[\begin{array}{l}
d Y_{1} \\
d Y_{2}
\end{array}\right]=\left[\begin{array}{c}
L+L^{\sharp} \\
-i\left(L-L^{\sharp}\right)
\end{array}\right] d t+\left[\begin{array}{l}
d W_{1} \\
d W_{2}
\end{array}\right] .
$$

In this work we focus on open harmonic oscillators. The dynamics of each oscillator are described by $\mathrm{t}$ wo self-adjoint operators position $q_{j}$ and momentum $p_{k}$, which satisfy the canonical commutation relations $\left[q_{j}, p_{k}\right]=2 i \delta_{j k}$ where $\delta_{j k}$ is the Kronecker delta. It is convenient to collect the position and momentum operators of the oscillators into an $n_{x^{-}}$ dimensional column vector $x(t)$, defined by $x(t)=$ $\left(q_{1}(t), p_{1}(t), q_{2}(t), p_{2}(t), \ldots, q_{\frac{n_{x}}{2}}(t), p_{\frac{n_{x}}{2}}(t)\right)^{T} \cdot \quad$ In this case the commutation relations can be re-written as:

$$
x(t) x(t)^{T}-\left(x(t) x(t)^{T}\right)^{T}=2 i \Theta_{n_{x}}
$$

where $\Theta_{n_{x}}=I_{\frac{n_{x}}{2}} \otimes J$ with $J=\left[\begin{array}{cc}0 & 1 \\ -1 & 0\end{array}\right]$. In general, $\Theta_{n}=I_{\frac{n}{2}} \otimes J$ for any even number $n \in \mathbb{N}$.
Harmonic oscillators, in particular, are defined by having a quadratic Hamiltonian of the form $\mathcal{H}=\frac{1}{2} x^{T} R x$ with $R$ being a $\mathbb{R}^{n_{x} \times n_{x}}$ symmetric matrix, and a coupling operator of the form $L=\Xi x$ with $\Xi$ being a $\mathbb{C}^{\frac{n_{w}}{2} \times n_{x}}$ matrix (here $n_{x}, n_{w}$ and $n_{y}$ are positive even numbers). A special property of open harmonic oscillators is that the differential equations governing $x(t)$ are linear. If we use an $n_{y}$-dimensional column vector $y(t)$ to incorporate all the quadratures of the output fields then, based on (3) and (4), the dynamics of a set of open harmonic oscillators can be described by the following linear QSDEs (James et al. (2008)):

$$
\begin{aligned}
& d x(t)=A x(t) d t+B d w(t) \\
& d y(t)=C x(t) d t+D d w(t)
\end{aligned}
$$

where $A, B, C$ are $\mathbb{R}^{n_{x} \times n_{x}}, \mathbb{R}^{n_{x} \times n_{w}}$ and $\mathbb{R}^{n_{y} \times n_{x}}$ matrices respectively, and $D=\left[\begin{array}{ll}I_{n_{y}} & 0_{n_{y} \times\left(n_{w}-n_{y}\right)}\end{array}\right]$. Furthermore, the coefficient matrices $A, B, C$ and $D$ satisfy the following physical realisability conditions (e.g., see James et al. (2008)):

$$
\begin{aligned}
& A \Theta_{n_{x}}+\Theta_{n_{x}} A^{T}+B \Theta_{n_{w}} B^{T}=0 \\
& B D^{T}=\Theta_{n_{x}} C^{T} \Theta_{n_{y}}
\end{aligned}
$$

These algebraic constraints on the coefficient matrices $A, B, C$ and $D$, independent of $\mathcal{H}$ and $L$, must be satisfied for (6) to correspond to a physically realisable quantum system. They were originally derived by requiring the canonical commutation relations of $x(t)(y(t))$ must hold for all times, a property enjoyed by open physical systems undergoing an overall unitary evolution (Gardiner and Zoller (2000), James et al. (2008)).

\section{Coherent quantum observers}

The structure of a coherent quantum observer is analogous to that of a classical Luenberger observer aimed at providing estimates of plant variables. In the physics literature, quantum filters are used to estimate quantum states using weak measurements. However, as for the quantum observer proposed in this paper, we consider exponentially tracking observables (not states) of a quantum plant in the sense of mean values in the Heisenberg picture free of any measurement.

\subsection{Existence of a coherent quantum observer}

A quantum observer is designed to track the corresponding quantum stochastic system (6). The quantum observer in this paper is of the form (Miao and James 
$(2012))$

$$
\begin{aligned}
& d \hat{x}(t)=(A-K C) \hat{x}(t) d t+K d y(t)+B_{0} d w_{0}(t), \\
& d \hat{y}(t)=\hat{C} \hat{x}(t) d t+\hat{D}\left[d y(t)^{T} d w_{0}(t)^{T}\right]^{T}
\end{aligned}
$$

where the $n_{x}$-dimensional column vector $\hat{x}(t)$ denotes the "estimate" of $x(t)$, and $K, B_{0}, \hat{C}$ are $\mathbb{R}^{n_{x} \times n_{y}}$, $\mathbb{R}^{n_{x} \times n_{w_{0}}}, \mathbb{R}^{n_{\hat{y}} \times n_{x}}$ matrices respectively. $\hat{D}$ is given by $\hat{D}=\left[\begin{array}{ll}I_{n_{\hat{y}}} & 0_{n_{\hat{y}} \times\left(n_{\hat{w}}-n_{\hat{y}}\right)}\end{array}\right]\left(\right.$ here $\left.n_{\hat{w}}=n_{y}+n_{w_{0}}\right) \cdot w_{0}(t)$ denotes an $n_{w_{0}}$-dimensional vector of self-adjoint additional quantum noises input to (8). Since the equations (8) characterising a coherent observer should correspond to the dynamics of an open quantum harmonic oscillator, the additional quantum noise signal $w_{0}(t)$ may be needed, for one has to ensure that the Heisenberg uncertainty principle is not violated and the commutation relations are preserved. The need to include additional quantum noise is well known in quantum optics (Hamerly and Mabuchi (2013), Wiseman and Milburn (2010)); for example, if one wishes to split an optical beam into two output beams, then additional quantum noise is required to ensure that the commutation relations are preserved, thus ensuring that the beam splitter is correctly described in quantum mechanics.

By comparing (6) and (8), we can obtain

$$
\begin{aligned}
d e(t)= & (A-K C) e(t) d t \\
& +(B-K D) d w(t)-B_{0} d w_{0}(t),
\end{aligned}
$$

where $e(t)=x(t)-\hat{x}(t)$ denotes the difference between the plant variables $x(t)$ and the corresponding observer variables $\hat{x}(t)$. Hence, the mean value of $e(t)(\bar{e}(t)=$ $\langle e(t)\rangle)$ satisfies

$$
\dot{\bar{e}}(t)=(A-K C) \bar{e}(t) .
$$

Therefore, $\bar{e}(t)$ will converge to zero asymptotically if and only if $(A-K C)$ is Hurwitz. That is, in the sense of mean values, this quantum observer can be used to track the corresponding quantum plant exponentially by appropriate choice of the observer gain $K$, and this convergence is independent of any additional quantum noise in the observer.

We know that the observer error covariance matrix $\bar{\Sigma}(t)=\frac{1}{2}\left\langle e(t) e^{T}(t)+\left(e(t) e^{T}(t)\right)^{T}\right\rangle$ evolves as

$$
\begin{aligned}
\dot{\bar{\Sigma}}(t)= & (A-K C) \bar{\Sigma}(t)+\bar{\Sigma}(t)(A-K C)^{T} \\
& +(K D-B)(K D-B)^{T}+B_{0} B_{0}^{T} .
\end{aligned}
$$

Since $(A-K C)$ is Hurwitz, standard results on Lyapunov equations give us $\lim _{t \rightarrow \infty} \bar{\Sigma}(t)=\bar{\Sigma}$, where $\bar{\Sigma}$ is the unique symmetric positive definite solution of the Lyapunov equation:

$$
\begin{aligned}
& (A-K C) \bar{\Sigma}+\bar{\Sigma}(A-K C)^{T} \\
& +(K D-B)(K D-B)^{T}+B_{0} B_{0}^{T}=0 .
\end{aligned}
$$

Furthermore, the steady-state mean squared error is

$$
\bar{\sigma}=\limsup _{t_{f} \rightarrow \infty} \frac{1}{t_{f}} \int_{0}^{t_{f}}\left\langle e^{T}(t) e(t)\right\rangle d t=\operatorname{Tr}(\bar{\Sigma}) .
$$

The following theorem provides a lower bound for the mean squared estimation error arising from the quantum nature of the problem, which differs from the classical case.

Theorem 1 Given systems (6) and (8), the mean squared error

$$
\bar{\sigma}(t)=\left\langle e^{T}(t) e(t)\right\rangle=\operatorname{Tr}(\bar{\Sigma}(t))
$$

is bounded below by $2 n_{x}$, i.e.,

$$
\bar{\sigma}(t) \geqslant 2 n_{x} .
$$

Proof By using the Cauchy-Bunyakovskii-Schwarz inequality (Jackiw (1968)), we can obtain

$$
\begin{aligned}
& \left\langle\left(x_{2 k-1}-\hat{x}_{2 k-1}\right)^{2}\right\rangle\left\langle\left(x_{2 k}-\hat{x}_{2 k}\right)^{2}\right\rangle \\
& \geqslant\left|\left\langle\left(x_{2 k-1}-\hat{x}_{2 k-1}\right)\left(x_{2 k}-\hat{x}_{2 k}\right)\right\rangle\right|^{2} \\
& =\mid\left\langle\frac{\left\{x_{2 k-1}-\hat{x}_{2 k-1}, x_{2 k}-\hat{x}_{2 k}\right\}}{2}\right\rangle \\
& +\left.\left\langle i \frac{\left[x_{2 k-1}-\hat{x}_{2 k-1}, x_{2 k}-\hat{x}_{2 k}\right]}{2 i}\right\rangle\right|^{2} \\
& \geqslant\left|\left\langle\frac{\left[x_{2 k-1}-\hat{x}_{2 k-1}, x_{2 k}-\hat{x}_{2 k}\right]}{2 i}\right\rangle\right|^{2} \\
& =\left|\left\langle\frac{\left[x_{2 k-1}, x_{2 k}\right]+\left[\hat{x}_{2 k-1}, \hat{x}_{2 k}\right]}{2 i}\right\rangle\right|^{2} \\
& =\left|\left\langle\frac{2 i+2 i}{2 i}\right\rangle\right|^{2}=4
\end{aligned}
$$

where $k=1,2, \ldots, \frac{n_{x}}{2}$, and thus

$$
\begin{aligned}
\left\langle e^{T} e\right\rangle & =\sum_{k=1}^{\frac{n_{x}}{2}}\left\langle\left(x_{2 k-1}-\hat{x}_{2 k-1}\right)^{2}+\left(x_{2 k}-\hat{x}_{2 k}\right)^{2}\right\rangle \\
& \geqslant 2 \sum_{k=1}^{\frac{n_{x}}{2}} \sqrt{\left\langle\left(x_{2 k-1}-\hat{x}_{2 k-1}\right)^{2}\right\rangle\left\langle\left(x_{2 k}-\hat{x}_{2 k}\right)^{2}\right\rangle} \\
& =2 \times \frac{n_{x}}{2} \times \sqrt{4}=2 n_{x} .
\end{aligned}
$$


Therefore, the mean squared estimation error satisfies

$$
\bar{\sigma}(t) \geqslant 2 n_{x} .
$$

Now, we give the definition of a coherent quantum observer for linear stochastic quantum plants.

Definition 1 Given a system (6), a system (8) is a coherent quantum observer for (6) if

1 there exists $K$ such that $(A-K C)$ is Hurwitz;

2 the system (8) corresponds to an open quantum harmonic oscillator.

The existence and design of a coherent quantum observer is analysed in the following theorem.

Theorem 2 Assume $(A, C)$ is detectable. Then there always exists $\left(K, B_{0}\right)$ such that the system (8) is a coherent quantum observer for the system (6).

Proof Since $(A, C)$ is detectable, $A-K C$ can be made Hurwitz by appropriate choice of $K$.

The system (8) can be rewritten as

$$
\begin{aligned}
d \hat{x}(t)= & (A-K C) \hat{x}(t) d t \\
& +\left[\begin{array}{ll}
K & B_{0}
\end{array}\right]\left[d y(t)^{T} d w_{0}(t)^{T}\right]^{T}
\end{aligned}
$$

where

$$
d y(t) d y(t)^{T}=F_{n_{y}} d t
$$

Here,

$$
F_{n_{y}}=I_{n_{y}}+i \Theta_{n_{y}},
$$

and in general, $F_{n}=I_{n}+i \Theta_{n}$ for any even number $n \in \mathbb{N}$. Hence,

$$
\left[d y(t)^{T} d w_{0}(t)^{T}\right]^{T}\left[d y(t)^{T} d w_{0}(t)^{T}\right]=F_{n_{y}+n_{w_{0}}} d t
$$

It is required that the quantum observer be physical, and by applying (James et al., 2008, Theorem 3.4), we know the physical realisability conditions are

$$
\begin{aligned}
& (A-K C) \Theta_{n_{x}}+\Theta_{n_{x}}(A-K C)^{T} \\
& +K \Theta_{n_{y}} K^{T}+B_{0} \Theta_{n_{w_{0}}} B_{0}^{T}=0, \\
& {\left[K B_{0}\right] \hat{D}^{T}=\Theta_{n_{x}} \hat{C}^{T} \Theta_{n_{\hat{y}}} .}
\end{aligned}
$$

Using the result in James et al. (2008), $B_{0}$ can be described as follows

$$
B_{0}=2 i \Theta_{n_{x}}\left[-\Lambda_{0}^{\dagger} \Lambda_{0}^{T}\right] \Gamma_{n_{w_{0}}}
$$

where

$$
\Gamma_{n_{w_{0}}}=P_{n_{w_{0}}} I_{\frac{n_{w_{0}}}{2}} \otimes M
$$

and

$$
M=\frac{1}{2}\left[\begin{array}{cc}
1 & i \\
1 & -i
\end{array}\right] .
$$

Here we use the symbol $P_{2 m}(m \in \mathbb{N})$ to denote a $2 m \times 2 m$ permutation matrix. For a column vector $a=$ $\left[\begin{array}{llll}a_{1} & a_{2} & \cdots & a_{2 m}\end{array}\right]^{T}$,

$$
P_{2 m} a=\left[\begin{array}{llllllll}
a_{1} & a_{3} & \cdots & a_{2 m-1} & a_{2} & a_{4} & \cdots & a_{2 m}
\end{array}\right]^{T}
$$

with $P_{2 m} P_{2 m}^{T}=P_{2 m}^{T} P_{2 m}=I_{2 m}$.

We can further obtain that,

$$
\begin{aligned}
& B_{0} \Theta_{n_{w_{0}}} B_{0}^{T} \\
& =4 \Theta_{n_{x}}\left[-\Lambda_{0}^{\dagger} \Lambda_{0}^{T}\right] \Gamma_{n_{w_{0}}} \Theta_{n_{w_{0}}} \Gamma_{n_{w_{0}}}^{T}\left[\begin{array}{c}
-\Lambda_{0}^{*} \\
\Lambda_{0}
\end{array}\right] \Theta_{n_{x}} \\
& =-2 i \Theta_{n_{x}}\left[\begin{array}{ll}
-\Lambda_{0}^{\dagger} & \Lambda_{0}^{T}
\end{array}\right] P_{n_{w_{0}}} \Theta_{n_{w_{0}}} P_{n_{w_{0}}}^{T}\left[\begin{array}{c}
-\Lambda_{0}^{*} \\
\Lambda_{0}
\end{array}\right] \Theta_{n_{x}} \\
& =-2 i \Theta_{n_{x}}\left[-\Lambda_{0}^{\dagger} \Lambda_{0}^{T}\right]\left[\begin{array}{cc}
0 \frac{n_{w_{0}}}{2} & I_{\frac{n_{w_{0}}}{2}} \\
-\frac{I_{w_{0}}}{2} & 0 \frac{n_{w_{0}}}{2}
\end{array}\right]\left[\begin{array}{c}
-\Lambda_{0}^{*} \\
\Lambda_{0}
\end{array}\right] \Theta_{n_{x}} \\
& =-4 \Theta_{n_{x}} \Im\left(\Lambda_{0}^{\dagger} \Lambda_{0}\right) \Theta_{n_{x}} .
\end{aligned}
$$

Then, based on (9) and (13), it can be concluded that

$$
\begin{aligned}
\Im\left(\Lambda_{0}^{\dagger} \Lambda_{0}\right)= & -\frac{i}{4} \Theta_{n_{x}}(A-K C) \\
& -\frac{i}{4}(A-K C)^{T} \Theta_{n_{x}} \\
& +\frac{i}{4} \Theta_{n_{x}} K \Theta_{n_{y}} K^{T} \Theta_{n_{x}}
\end{aligned}
$$

Now $\Lambda_{0}$ can be determined as any $\frac{n_{w_{0}}}{2} \times n_{x}$ complex matrix such that

$$
\begin{aligned}
\Lambda_{0}^{\dagger} \Lambda_{0}= & -\frac{i}{4} \Theta_{n_{x}}(A-K C) \\
& -\frac{i}{4}(A-K C)^{T} \Theta_{n_{x}} \\
& +\frac{i}{4} \Theta_{n_{x}} K \Theta_{n_{y}} K^{T} \Theta_{n_{x}}+\Xi_{0}
\end{aligned}
$$

where $\Xi_{0}$ is any real symmetric $n_{x} \times n_{x}$ matrix such that the right hand side of (14) is nonnegative definite.

Finally from (10), we choose

$$
\hat{C}=\Theta_{n_{\hat{y}}} \hat{D}\left[K B_{0}\right]^{T} \Theta_{n_{x}}
$$


where $\hat{D}=\left[\begin{array}{ll}I_{n_{\hat{y}}} & 0_{n_{\hat{y}} \times\left(n_{\hat{w}}-n_{\hat{y}}\right)}\end{array}\right]$. The matrices $K, B_{0}, \hat{C}, \hat{D}$ now define the coherent quantum observer (8).

Therefore, we have proved the existence of a physically realisable quantum observer, and it can be designed following the above procedure.

Corollary 1 For an optical cavity as a quantum plant given by $(S, L, \mathcal{H})=\left(\hat{I}, \sqrt{k_{p}} a_{p}, \Delta_{p} a_{p}^{\star} a_{p}\right)$, another optical cavity given by $(S, L, \mathcal{H})=\left(\hat{I}, \sqrt{k_{o}} a_{o}, \Delta_{o} a_{o}^{\star} a_{o}\right)$ is a coherent quantum observer for the plant if and only if

$$
\begin{aligned}
\Delta_{o} & =\Delta_{p} \\
k_{o} & =(\sqrt{2}-1)^{2} k_{p} .
\end{aligned}
$$

Here, $k_{p}, k_{o}>0$ are the decay rates, and $\Delta_{p}, \Delta_{o}$ denote the detuning frequencies of the cavities respectively. $a_{p}\left(a_{p}^{\star}\right)$ and $a_{o}\left(a_{o}^{\star}\right)$ are the annihilation (creation) operators of the plant and the observer respectively.

Proof First, we can obtain the equivalent models (6) and (8) for the plant and observer described by linear QSDEs with coefficients

$$
\begin{aligned}
A & =\left[\begin{array}{cc}
-\frac{k_{p}}{2} & \Delta_{p} \\
-\Delta_{p} & -\frac{k_{p}}{2}
\end{array}\right], C=\left[\begin{array}{cc}
\sqrt{k_{p}} & 0 \\
0 & \sqrt{k_{p}}
\end{array}\right], \\
K & =\left[\begin{array}{cc}
-\sqrt{k_{o}} & 0 \\
0 & -\sqrt{k_{o}}
\end{array}\right] .
\end{aligned}
$$

Then in order to make the cavity given by $(S, L, \mathcal{H})=$ $\left(\hat{I}, \sqrt{k_{o}} a_{o}, \Delta_{o} a_{o}^{\star} a_{o}\right)$ a coherent observer for the plant, we require that

$$
\begin{aligned}
A-K C & =\left[\begin{array}{cc}
-\frac{k_{p}}{2}+\sqrt{k_{o} k_{p}} & \Delta_{p} \\
-\Delta_{p} & -\frac{k_{p}}{2}+\sqrt{k_{o} k_{p}}
\end{array}\right] \\
& =\left[\begin{array}{cc}
-\frac{k_{o}}{2} & \Delta_{o} \\
\Delta_{o} & -\frac{k_{o}}{2}
\end{array}\right]
\end{aligned}
$$

which leads to (15).

Remark 1 Note that if $A-K C$ is Hurwitz and $(A-K C) \Theta_{n_{x}}+\Theta_{n_{x}}(A-K C)^{T}+K \Theta_{n_{y}} K^{T}=0$, then it is not necessary to input the additional quantum noise signal $w_{0}$ in (8), i.e., we can simply choose $B_{0}=0$ in this case. For instance, in Corollary 1, the cavity as the coherent quantum observer does not involve additional quantum noise input.
3.2 A simplified coherent quantum observer for annihilation-operator linear quantum systems

Here the systems under consideration are described in terms of complex annihilation operators by QSDEs of the form

$$
\begin{aligned}
& d a(t)=F a(t) d t+G d \bar{w}(t) ; a(0)=a_{0}, \\
& d \varsigma(t)=H a(t) d t+d \bar{w}(t)
\end{aligned}
$$

where $F \in \mathbb{C}^{n \times n}, G \in \mathbb{C}^{n \times n_{\bar{w}}}, H \in \mathbb{C}^{n_{\varsigma} \times n}\left(n, n_{\bar{w}}, n_{\varsigma}\right.$ are positive integers). Here $a(t)=\left[a_{1}(t), \ldots a_{n}(t)\right]^{T}$ is a vector of annihilation operators on an underlying Hilbert space. The noise $\bar{w}(t)$ is a vector of quantum Wiener processes with Itô table (canonical case):

$$
d \bar{w}(t) d \bar{w}(t)^{\dagger}=I_{n_{\bar{w}}} d t
$$

$L=\Lambda a\left(\Lambda\right.$ is a complex $n_{\bar{w}} \times n$ matrix $)$ represents the coupling operator, and the Hamiltonian is given by $\mathcal{H}=$ $a^{\dagger} N a$ where $N$ is an $n \times n$ complex Hermitian matrix. Then $F, G$ and $H$ can be described in terms of $L$ and $\mathcal{H}$ as (see Maalouf and Petersen (2011)):

$$
F=-\left(i N+\frac{1}{2} \Lambda^{\dagger} \Lambda\right), G=-\Lambda^{\dagger}, H=\Lambda .
$$

In a similar way to the coherent quantum observers discussed previously, a quantum observer for annihilationoperator linear quantum systems can be written as:

$$
\begin{aligned}
& d r(t)=\hat{F} r(t) d t+K d \varsigma(t)+G_{0} d \bar{w}_{0}(t), \\
& d \zeta(t)=\hat{H} r(t) d t+\left[d \varsigma(t)^{T} d \bar{w}_{0}(t)^{T}\right]^{T}
\end{aligned}
$$

where $K \in \mathbb{C}^{n \times n_{\varsigma}}, G_{0} \in \mathbb{C}^{n \times n_{\bar{w}_{0}}}, \hat{H} \in \mathbb{C}^{n_{\bar{\varsigma}} \times n}, \hat{\Lambda} \in$ $\mathbb{C}^{\left(n_{\varsigma}+n_{\bar{w}_{0}}\right) \times n}, \hat{N} \in \mathbb{C}^{n \times n}$ (Hermitian). The coupling and Hamiltonian operators are given as $\hat{L}=\hat{\Lambda} r, \hat{\mathcal{H}}=r^{\dagger} \hat{N} r$ respectively. Also, $\hat{F}=F-K H=-\left(i \hat{N}+\frac{1}{2} \hat{\Lambda}^{\dagger} \hat{\Lambda}\right)$, $\hat{G}=\left[K G_{0}\right]=-\hat{\Lambda}^{\dagger}$ and $\hat{H}=\hat{\Lambda}^{\dagger}$. The corresponding condition for physical realizability is (see Maalouf and Petersen (2011))

$$
\hat{F}+\hat{F}^{\dagger}+\hat{G} \hat{G}^{\dagger}=0 .
$$

Theorem 3 A simplified coherent quantum observer (18) for an annihilation-operator linear quantum stochastic system (17) is given by

$$
\begin{aligned}
& \hat{\Lambda}=-\left[\begin{array}{c}
\lambda_{K} T_{K} \Lambda+\Lambda \\
\lambda_{G_{0}} T_{G_{0}} \Lambda
\end{array}\right], \\
& \hat{N}=N-\frac{i}{2} \lambda_{K} \Lambda^{\dagger} T_{K}^{\dagger} \Lambda+\frac{i}{2} \lambda_{K} \Lambda^{\dagger} T_{K} \Lambda
\end{aligned}
$$


when

$$
\Upsilon=-i N-\frac{3}{2} \Lambda^{\dagger} \Lambda-\lambda_{K} \Lambda^{\dagger} T_{K}^{\dagger} \Lambda
$$

is Hurwitz.

Here, $T_{K} \in \mathbb{C}^{n_{\varsigma} \times n_{\varsigma}}$ and $T_{G_{0}} \in \mathbb{C}^{n_{w_{0}} \times n_{w_{0}}}$ are arbitrarily unitary matrices. $\lambda_{K}$ is a positive real parameter while $\lambda_{G_{0}}$ is non-negative real and $\lambda_{K}^{2}+\lambda_{G_{0}}^{2}=2$.

Also, if $\lambda_{K}=\sqrt{2}$, no additional quantum noise input $\bar{w}(t)$ to the observer is required.

Proof Note that

$$
\begin{aligned}
\hat{F} & =F-K H=-\left(i N+\frac{1}{2} \Lambda^{\dagger} \Lambda\right)-K \Lambda, \\
\hat{F}^{\dagger} & =-\left(-i N+\frac{1}{2} \Lambda^{\dagger} \Lambda\right)-\Lambda^{\dagger} K^{\dagger}, \\
\hat{G} \hat{G}^{\dagger} & =\left[\begin{array}{ll}
K & G_{0}
\end{array}\right]\left[\begin{array}{c}
K^{\dagger} \\
G_{0}^{\dagger}
\end{array}\right]=K K^{\dagger}+G_{0} G_{0}^{\dagger} .
\end{aligned}
$$

Since the observer (18) is required to be physically realisable, we must have

$$
\begin{aligned}
& \hat{F}+\hat{F}^{\dagger}+\hat{G} \hat{G}^{\dagger} \\
& =-K \Lambda-\Lambda^{\dagger} K^{\dagger}-\Lambda^{\dagger} \Lambda+K^{\dagger} K+G_{0} G_{0}^{\dagger} \\
& =0
\end{aligned}
$$

which is equivalent to

$$
\left(K-\Lambda^{\dagger}\right)\left(K^{\dagger}-\Lambda\right)+G_{0} G_{0}^{\dagger}=2 \Lambda^{\dagger} \Lambda .
$$

It is obvious that

$$
K=\lambda_{K} \Lambda^{\dagger} T_{K}^{\dagger}+\Lambda^{\dagger}, G_{0}=\lambda_{G_{0}} \Lambda^{\dagger} T_{G_{0}}^{\dagger}
$$

is an explicit solution to (21).

Here $T_{K} \in \mathbb{C}^{n_{\varsigma} \times n_{\varsigma}}$ and $T_{G_{0}} \in \mathbb{C}^{n_{w_{0}} \times n_{w_{0}}}$ are arbitrarily unitary matrices. $\lambda_{K}$ is a positive real parameter while $\lambda_{G_{0}}$ is non-negative real and $\lambda_{K}^{2}+\lambda_{G_{0}}^{2}=2$.

$\lambda_{K}=\sqrt{2}$ and $\lambda_{G_{0}}=0$ indicates that no additional quantum noise input to the observer is required.

Therefore, (19)-(20) determine a simplified quantum observer (18) for the annihilation operator linear quantum system (17) if

$$
\Upsilon=F-K H=-i N-\frac{3}{2} \Lambda^{\dagger} \Lambda-\lambda_{K} \Lambda^{\dagger} T_{K}^{\dagger} \Lambda
$$

is Hurwitz.

\section{Quantum correlations in joint plant-observer Gaussian quantum systems}

Entanglement, a unique feature of quantum systems, is central to all branches of the emerging field of quantum information and quantum computation (Nielsen and Chuang (2000)). Quantum systems can become entangled through various types of interactions, and because there is quantum information flowing into the corresponding coherent quantum observer from a quantum plant, it is not surprising that entanglement can be generated in this situation. The Peres-Horodecki PPT criterion is necessary and sufficient for separability of two-mode Gaussian states, and more generally, of all $(1+n)$-mode Gaussian states under $1 \times n$ bipartitions (Pirandola et al. (2009)). In this section, we explore the quantum correlations in a joint plant-observer quantum system. Measuring Gaussian entanglement is carried out using the covariance matrix, which is closely linked to the structure of a coherent quantum observer. As a result, it is critical to choose appropriate $K$ and $B_{0}$ in (8) so that the joint plant-observer quantum system is entangled. Furthermore, the underlying quantum states may be inferred via the interplay between the Heisenberg and Schrödinger pictures (Pan et al. (2014)).

Without loss of generality, we consider two-mode linear quantum stochastic systems, i.e. both the quantum plant (6) and the corresponding coherent quantum observer (8) have a single degree of freedom, which are initialised in Gaussian states.

The joint system, which is a cascade of a linear Gaussian quantum system and the corresponding coherent quantum observer, is of the form:

$$
d \tilde{x}(t)=\tilde{A} \tilde{x}(t) d t+\tilde{B} d \tilde{w}(t)
$$

where

$$
\begin{aligned}
& \tilde{x}(t)=\left(x^{T}(t), \hat{x}^{T}(t)\right)^{T}, \tilde{w}(t)=\left(w^{T}(t), w_{0}^{T}(t)\right)^{T}, \\
& \tilde{A}=\left[\begin{array}{cc}
A & 0 \\
K C & A-K C
\end{array}\right], \tilde{B}=\left[\begin{array}{cc}
B & 0 \\
K D & B_{0}
\end{array}\right] .
\end{aligned}
$$

For a system of the form (22), the symmetric covariance matrix is defined by

$P(t)=\frac{1}{2}\left\langle\tilde{x}(t) \tilde{x}^{T}(t)+\left(\tilde{x}(t) \tilde{x}^{T}(t)\right)^{T}\right\rangle-\langle\tilde{x}(t)\rangle\left\langle\tilde{x}^{T}(t)\right\rangle$

and satisfies the differential equation

$$
\dot{P}(t)=\tilde{A} P(t)+P(t) \tilde{A}^{T}+\tilde{B} \tilde{B}^{T}, P(0)=P_{0} .
$$

Assume $\tilde{A}$ is Hurwitz, then the steady-state symmetrised covariance matrix $P=\lim _{t \rightarrow \infty} P(t)$ satisfies the real 
Lyapunov equation (Nurdin et al. (2012),Wiseman and Milburn (2010))

$$
\tilde{A} P+P \tilde{A}^{T}+\tilde{B} \tilde{B}^{T}=0 .
$$

It is of much importance to see the differences between tracking systems with classical and quantum components, and thus one may expect to generate entanglement in a joint plant-observer quantum system. The following theorem gives us an indication about how to choose the observer gain $K$ and $B_{0}$ so that a plant and the corresponding observer become entangled. We use $\rho(t)$ to represent the density operator at time $t \geqslant 0$ of the joint plant-observer quantum system. $\rho(0)$ is the initial density operator, and $\rho(0)=\rho_{\text {sys }}(0) \otimes \rho_{\text {obs }}(0)$ with $\rho_{\text {sys }}(0)$ and $\rho_{\text {obs }}(0)$ denoting the initial density operators of the plant and the observer respectively. Also, the joint quantum system we consider is initialised in a Gaussian state, namely $\rho(0)$ is Gaussian (Nurdin et al. (2012)).

Theorem 4 Assume a one-mode quantum plant of the form (6), and the joint system (22) is Gaussian and initially separable with $\tilde{A}$ being Hurwitz. If $i K \Theta_{n_{y}} K^{T}$ is negative semidefinite, then $\rho(t)$ is separable for all $t \geqslant 0$.

Proof First, we point out that

$$
\Theta_{n_{w}} D^{T}=\left[\begin{array}{c}
\Theta_{n_{y}} \\
0_{\left(n_{w}-n_{y}\right) \times n_{y}}
\end{array}\right]=D^{T} \Theta_{n_{y}} .
$$

Since the one-mode quantum plant is physically realisable, one must have

$$
A J+J A^{T}+B \Theta_{n_{w}} B^{T}=0,
$$

and

$$
B D^{T}=J C^{T} \Theta_{n_{y}} .
$$

Equation (26) is equivalent to

$$
B D^{T} \Theta_{n_{y}}=-J C^{T},
$$

and by using (25), we obtain

$$
B \Theta_{n_{w}} D^{T}=-J C^{T} .
$$

Similarly, because of the physical realisability of the observer, we know

$$
\begin{aligned}
& (A-K C) J+J(A-K C)^{T} \\
& +K \Theta_{n_{y}} K^{T}+B_{0} \Theta_{n_{w_{0}}} B_{0}^{T}=0 .
\end{aligned}
$$

In order to apply (Nurdin et al., 2012, Theorem 4), we let $\tilde{\Theta}_{2}=\operatorname{diag}(J,-J), \tilde{\Theta}_{n_{w}}=\operatorname{diag}\left(\Theta_{n_{w}},-\Theta_{n_{w_{0}}}\right)$ and
$\tilde{P}=P+i \tilde{\Theta}_{2}$. Then

$$
\tilde{A} \tilde{\Theta}_{2}+\tilde{\Theta}_{2} \tilde{A}^{T}+\tilde{B} \tilde{\Theta}_{n_{w}} \tilde{B}^{T}=\left[\begin{array}{cc}
0 & 0 \\
0 & 2 K \Theta_{n_{y}} K^{T}
\end{array}\right],
$$

and thus

$$
i \tilde{A} \tilde{\Theta}_{2}+i \tilde{\Theta}_{2} \tilde{A}^{T}+i \tilde{B} \tilde{\Theta}_{n_{w}} \tilde{B}^{T}+F(K)=0,
$$

where

$$
F(K)=\left[\begin{array}{cc}
0 & 0 \\
0 & -2 i K \Theta_{n_{y}} K^{T}
\end{array}\right] \geqslant 0
$$

By adding (24) to (27), we obtain

$$
\tilde{A} \tilde{P}(t)+\tilde{P}(t) \tilde{A}^{T}+\tilde{B} \tilde{F}_{n_{w}} \tilde{B}^{T}+F(K)=\dot{\tilde{P}}(t)
$$

where $\tilde{F}_{n_{w}}=\operatorname{diag}\left(F_{n_{w}}, F_{n_{w_{0}}}^{\sharp}\right)$.

Since the joint Gaussian system is initially separable, $\tilde{P}(0) \geqslant 0$ holds. Meanwhile, as $\tilde{A}$ is Hurwitz, $F(K) \geqslant 0$ and $\tilde{F}_{n_{w}} \geqslant 0$, then $\tilde{P}(t) \geqslant 0$ for all $t \geqslant 0$. Therefore, we can conclude that the joint steady state $\rho(t)$ is separable for all times if $i K \Theta_{n_{y}} K^{T}$ is negative semidefinite, otherwise $\rho(t)$ can be entangled.

Several recent studies have shown that separable states, traditionally referred to as "classically correlated", might retain a signature of quantumness with potential operational applications for quantum technology. One such signature is the quantum discord, which aims to capture all the quantum correlations in a bipartite state, including but not restricted to entanglement (Freitas and Paz (2012)). The quantum discord is investigated in this paper, and we check whether the Gaussian quantum discord $D(\rho)$ is non-zero even if a joint plant-observer system turns out to be separable. If $D(\rho)$ is positive, the joint system possesses some quantumness (e.g., see Freitas and Paz (2012) and references therein). Also, in the following example, the smaller symplectic eigenvalue $\nu_{-}(t)$ of the partially transposed state is calculated to quantify the entanglement. Considering two-mode joint plant-observer systems, a joint Gaussian state is entangled at $t$ if and only if $\nu_{-}(t)<1$, otherwise it is separable (Pirandola et al. (2009), Nurdin et al. (2012), Yamamoto et al. (2008)). The purity $\mu(t)$ of a Gaussian state can be tracked as well.

Example 1 We consider an optical parametric oscillator as follows

$$
\begin{aligned}
d x & =\left[\begin{array}{cc}
-0.4 & 0 \\
0 & -0.6
\end{array}\right] x d t-d w \\
d y & =x d t+d w
\end{aligned}
$$


The system coefficients of the coherent quantum observer are chosen as $\left(K, B_{0}\right)=\left(I_{2} ;\left[\begin{array}{ll}1 & 0 \\ 0 & 0\end{array}\right],\left[\begin{array}{ll}1 & 0 \\ 0 & 2\end{array}\right]\right)$.

The initial covariance matrix of the joint plant-observer system is $P(0)=2 I_{4}$ corresponding to a Gaussian separable mixed state.

It can be verified that the coherent quantum observer with $\left(K, B_{0}\right)$ given above of the form (8) is physically realisable, i.e., (9)-(10) are satisfied.
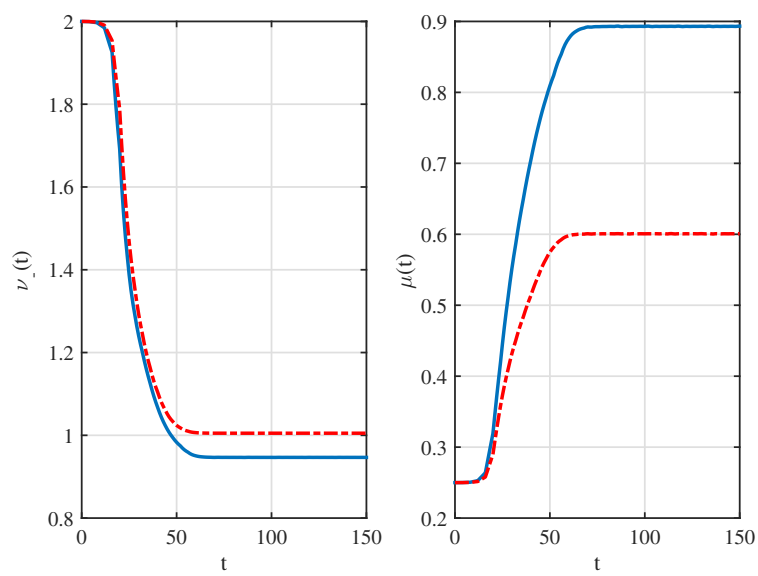

Fig. 2. The evolution of $\nu_{-}(t)$ and $\mu(t)$ starting from a separable Gaussian state over time, with the solid line and the dashed line corresponding to different $K$ values.

The evolution of $\nu_{-}(t)$ and $\mu(t)$ over time is shown in Fig. 2. The dashed lines show the evolution until steady state when $K$ is singular. It can be seen that $\nu_{-}(t) \geqslant 1$ for all times. Therefore, the joint state remains separable if $K$ is not invertible.

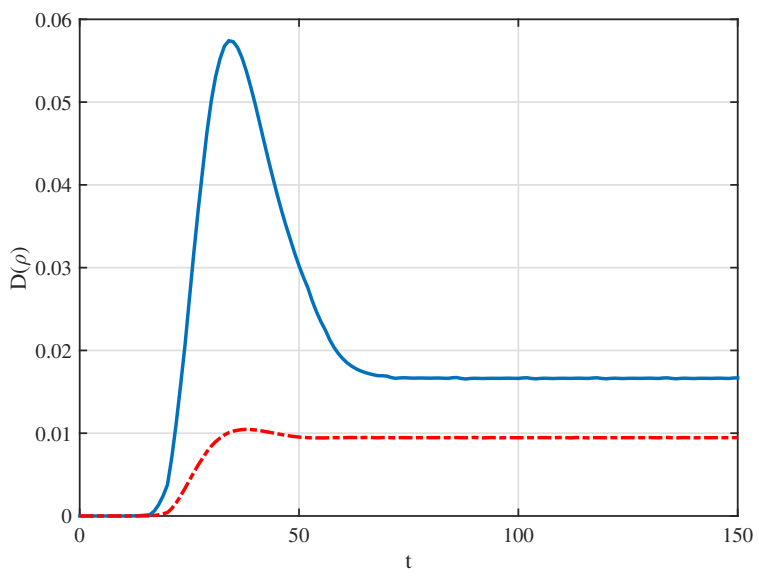

Fig. 3. The evolution of $D(\rho)$ starting from a separable Gaussian state over time, with the solid line and the dashed line corresponding to different $K$ values.
The solid lines in Fig. 2 correspond to the evolution of $\nu_{-}(t)$ and $\mu(t)$ when $K=I_{2}$. This clearly illustrates that the joint state becomes entangled subsequently since the output of the quantum plant (28) (a vector of non-commutative quantum signals) is fed to the coherent quantum observer. After a period of time, the joint quantum plant-observer system converges to a mixed steady state and remains entangled.

As shown in Fig. 3, after a certain period of time, the values of $D(\rho)$ are not zero in both cases, which indicates there exists quantumness in the joint plant-observer system even if the joint state is separable when $K$ is singular (dashed line). This can be understood by considering that the states $\rho_{\text {sys }}(t)$ and $\rho_{\text {obs }}(t)$ are not physically distinguishable, and thus not all the information about them can then be locally retrieved.

\section{Discussion and conclusion}

We have constructed a class of coherent quantum observers which can track linear quantum stochastic systems asymptotically in the sense of mean values, and a lower bound for the mean squared observer error is provided due to the quantum nature. We prove the existence of such a coherent quantum observer, and show how to determine the observer parameters explicitly. Some properties of the quantum observer have been given, for example, the covariance bound, as well as entanglement and quantum discord. In particular, Example 1 demonstrates that different coefficient matrices of a coherent quantum observer play a vital role in the occurrence of entanglement in joint states considering a two-mode Gaussian quantum system, and therefore it is critical to choose appropriate $\left(K, B_{0}\right)$ so as to render the joint states entangled. Future work involves using coherent quantum observers for coherent feedback design.

\section{References}

Anderson, B. D. O. and J. B. Moore (1979). Optimal Filtering. Englewood Cliffs, NJ: Prentice-Hall.

Belavkin, V. P. (1994). Quantum diffusion and measurement and filtering. Theory of Probability and Its Applications 38(4), 573-585.

Bouten, L., R. van Handel, and M. R. James (2007). An introduction to quantum filtering. SIAM Journal on Control and Optimization 46(6), 2199-2241.

Carmichael, H. J. (1993). Quantum trajectory theory for cascaded open systems. Physical Review Letters 70(15), 2273-2276.

Ellis, G. (2002). Observers in Control Systems. San Diego, USA: Academic Press.

Freitas, J. N. and J. P. Paz (2012). Dynamics of Gaussian discord between two oscillators interacting with a common environment. Physical Review A 85(3), 032118 . 
Gardiner, C. and P. Zoller (2000). Quantum Noise: A Handbook of Markovian and Non-Markovian Quantum Stochastic Methods With Applications to Quantum Optics. Springer Series in Synergetics. New York: Springer.

Gough, J. and M. R. James (2009). The series product and its application to quantum feedforward and feedback networks. IEEE Transactions on Automatic Control 54(11), 2530-2544.

Hamerly, R. and H. Mabuchi (2013). Coherent controllers for optical-feedback cooling of quantum oscillators. Physical Review A 87(1), 013815.

Jackiw, R. (1968). Minimum uncertainty product, number-phase uncertainty product, and coherent states. Journal of Mathematical Physics 9(3), 339-346.

James, M. R., H. I. Nurdin, and I. R. Petersen (2008). $\mathrm{H}^{\infty}$ control of linear quantum stochastic systems. IEEE Transactions on Automatic Control 53(8), 1787-1803.

Luenberger, D. G. (1966). Observers for multivariable systems. IEEE Transactions on Automatic Control 11, 190-199.

Maalouf, A. I. and I. R. Petersen (2011). Bounded real properties for a class of annihilation-operator linear quantum systems. IEEE Transactions on Automatic Control 56(4), 786-801.

Miao, Z., M. R. Hush, and M. R. James (2015). Coherently tracking the covariance matrix of an open quantum system. Physical Review A 92(1), 012115.

Miao, Z. and M. R. James (2012). Quantum observer for linear quantum stochastic systems. In Proceedings of 51st IEEE Conference on Decision and Control, Maui, Hawaii, pp. 1680-1684.

Miao, Z., M. R. James, and V. Ugrinovskii (2015). Pole placement design for quantum systems via coherent observers. In Proceedings of 54th IEEE Conference on Decision and Control, Osaka, Japan, pp. 5784-5789.

Nielsen, M. A. and I. L. Chuang (2000). Quantum Computation and Quantum Information. Cambridge, UK: Cambridge University Press.

Nurdin, H. I., I. R. Petersen, and M. R. James (2012). On the infeasibility of entanglement generation in Gaussian quantum systems via classical control. IEEE Transactions on Automatic Control 57(1), 198-203.

Pan, Y., H. Amini, Z. Miao, J. Gough, V. Ugrinovskii, and M. R. James (2014). Heisenberg picture approach to the stability of quantum markov systems. Journal of Mathematical Physics 55(6), 062701.

Parthasarathy, K. R. (1992). An Introduction to Quantum Stochastic Calculus. Modern Birkhäuser Classics. Berlin, Germany: Birkhauser.

Pirandola, S., A. Serafini, and S. Lloyd (2009). Correlation matrices of two-mode bosonic systems. Physical Review A 79(5), 052327.

Wiseman, H. M. and G. J. Milburn (2010). Quantum Measurement and Control. Cambridge, UK: Cambridge Univ. Press.

Yamamoto, N. (2006). Robust observer for uncertain linear quantum systems. Physical Review A 74(3),
032107.

Yamamoto, N., H. I. Nurdin, M. R. James, and I. R. Petersen (2008). Avoiding entanglement sudden death via measurement feedback control in a quantum network. Physical Review A 78(4), 042339. 